\title{
CATEGORIZAÇÃO DOS PROGRAMAS DE CAPACITAÇÃO DAS MAIORES COMPANHIAS BRASILEIRAS DE CAPITALABERTO
}

\section{CATEGORIZTION OF TRAINING PROGRAMS OF LARGEST PUBLICLY LISTED BRAZILIANS COMPANIES}

Anna Beatriz Grangeiro Ribeiro Maia

Universidade Federal do Ceará - Brasil.

Universidade de Fortaleza - Brasil

abgrmaia@gmail.com

ORCID: https://orcid.org/0000-0003-2402-1546

Camila Oliveira Barbosa

Universidade Federal do Ceará - Brasil.

camila.o.b1990@gmail.com

ORCID: https://orcid.org/0000-0002-6557-9282

Alessandra Carvalho de Vasconcelos

Universidade Federal do Ceará - Brasil.

alevasconcelos.ufc@gmail.com

ORCID: https://orcid.org/0000-0002-6480-5620
Victor Daniel Vasconcelos

Universidade Federal do Ceará - Brasil.

victordaniel.ufc@gmail.com

ORCID: https://orcid.org/0000-0002-1756-6241

\section{RESUMO}

O objetivo do estudo foi categorizar os programas de capacitação - enquanto práticas sustentáveis de gestão do capital humano. Oportunamente, identificaram-se os atributos organizacionais (institucionais e humanos) correlacionados ao relato de tais práticas nos relatórios de sustentabilidade, padrão GRI. Foram identificados 2.255 programas de capacitação, a partir da análise de conteúdo dos 104 relatórios de sustentabilidade, no biênio 2015-2016, de 6I companhias diferentes, listadas entre as maiores companhias abertas, segundo a revista Exame Melhores \& Maiores (2017). Os resultados da análise de conteúdo apontam a concentração de programas de capacitação à categoria Responsabilidade socioambiental (40\%). As análises de correlação indicaram, contra expectativas, ser incipiente à correlação entre o relato de programas de capacitação pelas empresas e os atributos organizacionais analisados, sendo mais evidente quando tratados por categoria. A principal contribuição deste estudo residiu na categorização dos programas de capacitação relatados pelas maiores empresas de capital aberto no Brasil.

\section{PALAVRAS-CHAVE}

Gestão do capital humano. Programas de capacitação. Atributos organizacionais. Relatórios de sustentabilidade. GRI. 


\section{ABSTRACT}

The purpose of this study was to categorize corporate training programs as sustainable practices of human resource management. In addition, we correlated organizational (institutional and human) attributes with the disclosure of such practices in sustainability reports adhering to GRI guidelines. Thus, 2,255 training programs were identified by content analysis of 104 sustainability reports for the 2015-2016 biennium, issued by $6 \mathrm{I}$ top Brazilian public firms, according to the ranking of Exame Melhores \& Maiores (2017). The content analysis revealed the predominance (40\%) of the category 'socienvironmental responsibility'. Contrary to our expectations, the correlation between the reporting of corporate training programs and organizational attributes (most evident when viewed by category) was merely embryonic. The main contribution of this study was the categorization of the training programs reported by top Brazilian public firms.

\section{KEYWORDS}

Human resource management. Training programs. Organizational attributes. Sustainability reports. GRI.

\section{INTRODUÇÃO}

As organizações buscam estratégias que garantam a sua continuidade, ante o caráter instável e desafiador dos mercados nacional e internacional. Diversos argumentos teóricos, a partir da Teoria do Capital Humano - TCH (SCHULTZ, 196I; POZAS; JAUREGUI, 20I2; KAROLCZAK; SOUZA, 20I7), da Teoria da Agência (UTRILLA; GRANDE-TORRALEJA; LORENZO, 20I5) e da Visão Baseada em Recursos - VBR (CAMPOS; SANTOS; RODRIGUES, 20I4), sugerem as práticas de gestão do capital humano como propiciadoras de vantagem competitiva sustentável, seja: por otimizar serviços e produção e dirimir problemas de agência (UTRILLA; GRANDE-TORRALEJA;LORENZO, 20I5); pela dificuldade de serem copiadas, imitadas ou adaptadas (CAMPOS; SANTOS; RODRIGUES, 20I4); pelo desenvolvimento de competências e habilidades profissionais; ou pela construção de uma cultura contínua de aprendizagem (ENGELEN et al., 20I5).

Não obstante a isso, como resposta à maior conscientização global para o papel das organizações na sociedade, existe uma demanda crescente para as maiores empresas do mundo demonstrarem compromisso com a sustentabilidade (EHNERT et al., 20I5). Neste contexto, um número crescente de organizações parece disposto a relatar o desempenho de sustentabilidade econômica, social e ambiental (SCHALTEGGER;WAGNER, 2006), o que é enfatizado pelo progresso nos padrões de relatórios como o Global Reporting Initiative (GRI).

Um avanço mais recente à agenda de sustentabilidade tem sido a Sustainable HRM, ou gestão de recursos humanos sustentável, que abrange um corpo crescente de literatura acadêmica e profissional conectando 
as noções de sustentabilidade corporativa às práticas de human resource management - HRM (EHNERT; HARRY; ZINK, 20I4; EHNERT et al., 2015; GUERCl et al., 2018; LELEBINA; GAND, 20I8; SHRM, 20I I). No geral, as principais funções da HRM são contribuir para o desenvolvimento de empresas sustentáveis de forma econômica, social e ambiental, e tornar os sistemas de gestão de capital humano mais sustentáveis (EHNERT; HARRY, 20I2; EHNERT et al., 20I4).

Operacionalmente, a gestão do capital humano busca fontes de vantagem competitiva de longo prazo, a partir de uma abordagem estratégica de sua função, enfatizando o investimento em capital humano, o envolvimento dos empregados e o alinhamento integrativo dos processos de gestão do trabalho com sistemas de recompensa baseados em benefício mútuo (SILVEIRA; MAESTRO FILHO, 20I3).

Nesta perspectiva, todos os processos que geram incentivos ao desenvolvimento do capital humano, como os programas de capacitação tratados neste estudo, podem gerar vantagens competitivas para a empresa. Para os fins desta pesquisa, é considerado programa de capacitação o desenvolvimento sistemático de conhecimentos, normas, conceitos ou atitudes que resulte em meIhoria do desempenho, tornando-se parte da aprendizagem e da mudança organizacional, da avaliação do empregado e do desenvolvimento de sua carreira (TASCA; ENSSLIN; ENSSLIN, 20I2).

Assim, ao levar em conta um conceito de programa de capacitação lato sensu, essa pesquisa apoia-se em um modelo específico de aprendizagem organizacional, que compreende treinamento, desenvolvimento humano, desenvolvimento por competência, programas de educação permanente, capacitação, palestras, cursos, workshops, coaching, mentoring e outras formas de transmissão de conhecimentos no âmbito organizacional.

Destaca-se que embora o capital humano tenha se revelado essencial para a sobrevivência e o crescimento organizacional, ainda há poucos estudos com foco em questões sobre as práticas de treinamento na América Latina (POZAS; JAUREGUI, 20I2).Aliado $\mathrm{a}$ isto, mesmo com o crescente número de trabalhos sobre Sustainable HRM nos últimos anos, o exame empírico dos relatos de sustentabilidade corporativa com enfoque na gestão de recursos humanos ainda é incipiente (EHNERT et al., 20I5). Destarte, este artigo preenche essa lacuna analisando os relatórios de sustentabilidade das maiores companhias abertas do Brasil, e tem como objetivo categorizar os programas de capacitação - enquanto práticas sustentáveis de gestão do capital humano. Adicionalmente, buscou-se identificar os atributos organizacionais (institucionais e humanos) correlacionados ao relato de tais práticas nos relatórios de sustentabilidade, padrão GRI.

Para tanto, a partir da aplicação da análise de conteúdo, foram identificados os programas de capacitação divulgados nos relatórios GRI de 2015 e 2016 da amostra, e, na sequência, a sua categorização. Os dados relativos aos atributos organizacionais considerados na pesquisa, especificamente voltados para a abordagem institucional e humana, foram extraídos dos formulários de referência e da base Economática. Os programas de capacitação relatados pelas empresas, de forma geral e por categoria, passaram por uma análise de correlação com os atributos institucionais e humanos identificados.

Assim como Ehnert et al. (20I5), este estudo se concentrou nas maiores empresas porque são reconhecidas como influentes na 
disseminação das melhores práticas por meio de sua posição no ápice das cadeias de valor globais (GEREFFI, 20I4). Vale ressaltar que este artigo não discorre sobre como o conceito teórico de Sustainable HRM se traduz em práticas que visam o comprometimento das pessoas com a estratégia organizacional, mas analisa os programas de capacitação enquanto práticas sustentáveis de gestão do capital humano - reportados no relato da sustentabilidade corporativa.

Considerando-se que o sistema de gestão de capital humano possui função estratégica na empresa (OLIVEIRA et al., 2017), a categorização dos programas de capacitação configura-se como uma eminente contribuição do estudo, somada à identificação de possíveis atributos relacionados com o seu relato, haja vista que podem auxiliar na construção de uma tradição de pesquisa cumulativa nesse campo. Em especial, os achados podem auxiliar as empresas a tomar decisões acerca de seus sistemas de gestão de pessoas, da divulgação voluntária sobre programas de capacitação, cujo disclosure, mesmo sendo voluntário no Brasil, é útil para se avaliar a perspectiva futura da empresa (FÉLIX JúNIOR et al., 2017).

\section{SUPORTE TEÓRICO E DESENVOL- VIMENTO DAS HIPÓTESES}

Diante das crescentes pressões por relatos de sustentabilidade $(\mathrm{HAHN}$; $\mathrm{KUH}$ NEN, 2013), as empresas reagem divulgando vários aspectos da sustentabilidade voluntariamente (KPMG, 20l7), visando maior transparência e accountability perante os stakeholders (ROBERTS, 2009). No entanto, diante da ausência de auditoria padronizada e obrigatória dos relatórios de sustentabilidade, é importante observar que a elaboração de relatórios apri- morados não significa necessariamente maior transparência. Conforme Roberts (2009), a empresa pode divulgar nesses relatórios informações irrelevantes para projetar uma imagem desejável, o que não significa que a empresa cumpriu com êxito sua responsabilidade socioambiental.

$\mathrm{Na}$ inexistência de quaisquer requisitos regulatórios, a GRI forneceu um conjunto de diretrizes que não apenas permitiu a comparação de práticas socioambientais entre empresas em todo o mundo, mas também desafiou o relato corporativo de uma série de práticas negativas (EHNERT et al., 20I5). De fato, as diretrizes da GRI são consideradas “o padrão global de fato" para relatórios de sustentabilidade das principais empresas globais (KPMG, 2017) e são usadas frequentemente por pesquisadores no campo dos relatos de sustentabilidade (EHNERT et al., 20 I5). A estrutura da GRI, modelo G4, fornece diretrizes em três categorias principais, refletindo ampla gama de questões dentro da sustentabilidade corporativa: econômica, ambiental e social. $A$ última categoria inclui, por sua vez, quatro subcategorias, dentre as quais duas estão relacionadas ao foco deste estudo: Práticas trabalhistas e trabalho decente e Direitos humanos.

Sob esse enfoque, Ehnert et al. (2015) analisaram os relatórios de sustentabilidade de 95 empresas da Forbes top 250 global companies e avaliaram comparativamente os aspectos de Sustainable HRM e os aspectos ambientais do gerenciamento sustentável. Adicionalmente, verificaram se os atributos organizacionais - especialmente o país de origem - influenciam os relatos das práticas sociais. Os resultados sugeriram que (i) a divulgação sobre o desempenho da gestão do capital humano não é inferior à do desempenho ambiental; (ii) as empresas relatam mais sobre sua força de trabalho interna do 
que sobre sua força de trabalho externa; e, (iii) diferenças internacionais não são tão aparentes quanto o esperado.

Uma vez exposto o contexto em que se insere esta pesquisa, descreve-se brevemente algumas obras alinhadas ao fenômeno em foco e que sustentam o estudo na construção das hipóteses levantadas.

Com base na TCH, Pozas e Jauregui (20I2), analisando 24 companhias peruanas, constataram um forte interesse no treinamento, em particular no desenvolvimento de competências, a partir de treinamento profissional. Verificou-se alta heterogeneidade de cursos ofertados pelas empresas, assim como de seus objetivos. Tal resultado pode ser confrontado a um dos pontos de análise desta pesquisa, quanto à categorização dos programas de capacitação das companhias brasileiras.

Utrilla et al. (20I5) pesquisaram os efeitos do coaching como prática de desenvolvimento de recursos humanos no desempenho de 498 empresas espanholas. A análise fatorial revelou impacto positivo do coaching no desempenho dos empregados e das empresas. Tais resultados apontaram para a possível correlação entre alguns atributos institucionais aqui investigados, a fim de compreender o fenômeno no Brasil.

Gaspar et al. (2016) identificaram as práticas de gestão do conhecimento mais utilizadas em indústrias brasileiras de software e as ferramentas de tecnologia da informação e comunicação a elas associadas. A categoria de práticas de gestão do conhecimento mais evidenciada foi a estratégia. Há de se ressaltar que as práticas tratadas isoladamente obtiveram um comportamento diverso das práticas agrupadas em dimensões. Isto sugere que as práticas de gestão do capital humano devem ser analisadas de forma geral e agrupadas, como proposto nesta pesquisa por meio da categorização dos programas.

Karolczak e Souza (2017) propuseram alguns aspectos relativos à formação, à qualificação, à motivação, à mobilidade profissional e às práticas de investimento em desenvolvimento da carreira como explicativos da escassez de profissionais de recursos humanos capacitados nas áreas de Ciências, Tecnologia, Engenharia, Matemática e afins. Tais aspectos foram utilizados nesta pesquisa para a construção do instrumento de categorização dos programas relatados pelas companhias brasileiras.

Outro estudo que levanta uma lacuna contemplada na presente pesquisa é o de Bortoluzzi, Genari e Macke (2017). Os autores caracterizaram a obsolescência e os aspectos vinculados à aquisição do capital humano em empresas de tecnologia. Segundo seus participantes, a aprendizagem formal, baseada no ensino superior, é a principal forma de aquisição do capital humano. Aqui, a partir da categorização dos programas de capacitação, analisa-se o relato da promoção das práticas de gestão de pessoas nas maiores companhias abertas brasileiras.

Fundamentado nas recomendações da literatura, que apontam a importância dos programas de capacitação, o estudo considera relevante categorizar os programas reportados pelas empresas brasileiras, aspecto não observado nos estudos empíricos sobre a temática. Ademais, foram formuladas duas hipóteses principais relativas aos potenciais atributos organizacionais correlacionados com o reporting de programas de capacitação, a saber: HI - Há correlação entre atributos institucionais e o relato de programas 
de capacitação; e H2 - Há correlação entre atributos humanos da alta gestão e o relato de programas de capacitação.

No tocante à hipótese $\mathrm{HI}$, à luz das sinalizações dos estudos apresentados no Quadro I, foram considerados sete atributos institucionais, cada um dos quais suportou uma hipótese operacional específica.

Quanto à hipótese $\mathrm{H} 2$, e com base nas recomendações dos estudos correlatos, também foram considerados sete atributos humanos da alta gestão, conforme exposto no Quadro 2.

Os estudos supramencionados exemplificam a forma como a temática vem sendo investigada na literatura, identificando-se, algumas vezes, dispersão e descontinuidade de pesquisas empíricas sobre programas de capacitação. Esse rol não é taxativo, porém possibilita uma visão das diversas óticas em que se manifesta esse construto. Além disso, comprova a originalidade desta pesquisa, ao estudar as maiores companhias abertas brasileiras, indicando uma categorização de seus programas, bem como identificando potenciais atributos correlacionados ao seu relato.

\section{MÉTODO}

O estudo descritivo investiga o relato de programas de capacitação, enquanto reporting

QUADRO 1 - Possíveis atributos institucionais correlacionados ao relato de programas de capacitação

\begin{tabular}{|c|l|l|c|}
\hline $\begin{array}{c}\text { Hipótese } \\
\text { operacional }\end{array}$ & Atributo institucional & \multicolumn{1}{|c|}{ Suporte teórico } & Resultado esperado \\
\hline H1a & Porte & Almeida e Aterido (2015) & + \\
\hline H1b & Rentabilidade & Utrilla e Grande-Torraleja (2012) & + \\
\hline H1c & Endividamento & Mamede, Nakamura, Nakamura, Jones e Jardim (2017) & - \\
\hline H1d & Setor & Gaspar et al. (2016), Hahn e Kühnen (2013) & + \\
\hline H1e & Idade & Assunção, De Luca e Vasconcelos (2017) & + \\
\hline H1f & Governança corporativa & Ambrozini (2017), Beuren e Marcello (2016) & + \\
\hline H1g & Internacionalização & Petry et al. (2014) & + \\
\hline
\end{tabular}

Fonte: Elaborado pelos autores.

QUADRO 2 - Possíveis atributos humanos correlacionados ao relato de programas de capacitação

\begin{tabular}{|c|l|l|c|}
\hline $\begin{array}{c}\text { Hipótese } \\
\text { operacional }\end{array}$ & \multicolumn{1}{|c|}{ Atributo humano } & \multicolumn{1}{|c|}{ Suporte teórico } & $\begin{array}{c}\text { Resultado } \\
\text { esperado }\end{array}$ \\
\hline $\mathrm{H} 2 \mathrm{a}$ & Número de conselheiros & Ambrozini (2017) & - \\
\hline $\mathrm{H} 2 \mathrm{~b}$ & Formação do chairman & $\begin{array}{l}\text { Barakat e Fischmann (2016), Manzaneque, Merino e Banegas } \\
(2011)\end{array}$ & + \\
\hline $\mathrm{H} 2 \mathrm{c}$ & HRM expertise dos conselheiros & Manzaneque, Merino e Banegas (2011) & + \\
\hline $\mathrm{H} 2 \mathrm{~d}$ & Independência do chairman & $\begin{array}{l}\text { Ambrozini (2017), Barakat e Fischmann (2016), Manzaneque, } \\
\text { Merino e Banegas (2011) }\end{array}$ & + \\
\hline $\mathrm{H} 2 \mathrm{e}$ & Número de diretores & Cortés, Echeverry, Ramiréz, Yangüe e Betancourt (2017) & - \\
\hline $\mathrm{H} 2 \mathrm{f}$ & $\begin{array}{l}\text { Formação do chief executive } \\
\text { officer (CEO) }\end{array}$ & $\begin{array}{l}\text { Carrasco (2011), Díez-De-Castro, Díez-Martín e Vázquez-Sá- } \\
\text { nche (2015), Mascena, Barakat e Fischmann (2016) }\end{array}$ & + \\
\hline $\mathrm{H} 2 \mathrm{~g}$ & HRM expertise dos diretores & $\begin{array}{l}\text { Carrasco (2011), Díez-De-Castro, Díez-Martín e Vázquez-Sán- } \\
\text { che (2015) }\end{array}$ & + \\
\hline
\end{tabular}

Fonte: Elaborado pelos autores. 
de pessoas em sustentabilidade, e possíveis atributos organizacionais correlacionados à sua narrativa. Para tanto, realizou-se uma pesquisa documental, por meio dos relatórios de sustentabilidade da GRI, dos formulários de referência e da base Economática.

A população reúne as 100 maiores companhias abertas brasileiras, por valor de mercado, segundo a revista Exame Melhores \& Maiores de 2017, sendo excluídas algumas empresas, por não divulgarem o relatório de sustentabilidade da GRI necessário para análise (Tabela I).

Conforme a Tabela I, a amostra reúne 104 empresas no biênio 20I5-2016, sendo 54 no ano de 2015 e 50 no ano de 2016, compreendendo $6 \mathrm{I}$ companhias diferentes.

$\mathrm{Na}$ sequência, procedeu-se à coleta das informações relativas aos programas de capacitação relatados pelas empresas, a partir da análise dos seus relatórios de 2015 e 2016, nos tópicos G4-LA9 e G4LA I0, contemplados na seção Treinamento e Educação, do modelo G4. Para a identificação e categorização dos programas de capacitação, utilizou-se a análise de conteúdo. Destaca-se que cada programa foi enquadrado exclusivamente dentro de uma das categorias e para garantir maior confiabilidade à categorização dos programas o enquadramento foi realizado, em um primeiro momento, por um dos pesquisadores e, posteriormente, revisado por outros dois pesquisadores simultaneamente.

Os dados relacionados aos atributos institucionais (Quadro 3) foram extraídos dos formulários de referência - Idade, Item 6.4; Governança corporativa, Item 18.6; e

TABELA 1 - Etapas de seleção da amostra do estudo

\begin{tabular}{c|l|c|c|c}
\hline \multirow{2}{*}{ Operação } & & \multicolumn{2}{c}{ Descrição } & \multicolumn{2}{c}{ Número de empresas } \\
\cline { 2 - 5 } & & $\mathbf{2 0 1 5}$ & $\mathbf{2 0 1 6}$ & Total \\
\hline$(=)$ & População & 100 & 100 & 200 \\
\hline$(-)$ & Empresas que não publicaram relatório de sustentabilidade & $(30)$ & $(41)$ & $(71)$ \\
\hline$(-)$ & Empresas que não publicaram relatório de sustentabilidade no padrão GRI & $(16)$ & $(9)$ & $(25)$ \\
\hline$(=)$ & Amostra & $\mathbf{5 4}$ & $\mathbf{5 0}$ & $\mathbf{1 0 4}$ \\
\hline
\end{tabular}

Fonte: Elaborada pelos autores.

QUADRO 3 - Atributos institucionais e respectiva operacionalização

\begin{tabular}{|l|l|}
\hline \multicolumn{1}{|c|}{ Atributos } & \multicolumn{1}{c|}{ Operacionalização } \\
\hline Porte & Valor do Ativo consolidado \\
\hline Rentabilidade & Lucro líquido consolidado \\
\hline Endividamento & Quociente entre o Passivo e o Patrimônio Líquido \\
\hline Setor & $\begin{array}{l}\text { Variável categórica, sendo atribuída maior numeração aos setores mais inovativos: } 1 \text { - Alimentos e } \\
\text { Bebidas; 2 - Comércio; 3 - Construção; 4 - Eletroeletrônicos; 5 - Energia Elétrica; } 6 \text { - Finanças e Segu- } \\
\text { ros; 7 - Máquinas Industriais; } 8 \text { - Mineração; } 9 \text { - Papel e Celulose; 10 - Petróleo e Gás; } 11 \text { - Química; } \\
12 \text { - Software e Dados; 13 - Telecomunicações; 14 - Transporte e Serviços; } 15 \text { - Veículos e Peças }\end{array}$ \\
\hline Idade & Em 2018, tomando-se por base o ano de constituição \\
\hline Governança corporativa & $\begin{array}{l}\text { Variável categórica, de acordo com o segmento de listagem, sendo atribuída maior numeração ao } \\
\text { mais alto nível diferenciado de governança corporativa: - - Tradicional; } 1 \text { - Nível 1; } 2 \text { - Nível 2; } 3 \text { - } \\
\text { Novo Mercado }\end{array}$ \\
\hline Internacionalização & Número de bolsas estrangeiras em que são negociadas as ações da empresa \\
\hline
\end{tabular}

Fonte: Elaborado pelos autores. 
Internacionalização, Item 6 - e da base Economática - Porte, Rentabilidade, Endividamento e Setor.

Os dados relacionados aos atributos humanos, extraídos dos formulários de referência (Itens 12 e 12.5/6), são apresentados no Quadro 4.

Concluída a coleta, obteve-se uma base totalizando I8.5 I9 informações, entre dados brutos e variáveis. Inicialmente, procedeu-se à preparação da matriz de entrada dos dados, isto é, verificaram-se os missing values. Para cada variável, os valores ausentes foram substituídos pela média, conforme adotado por Hahn, Scherer e Oliveira (2016).

A análise dos dados seguiu duas etapas principais atendendo aos objetivos do estudo: identificação dos programas de capacitação reportados nos relatórios e sua categorização; e, verificação dos atributos institucionais e humanos correlacionados ao relato de tais práticas.

$\mathrm{Na}$ primeira etapa, todos os programas de capacitação identificados nos 104 relatórios de sustentabilidade passaram pelos seguintes passos da análise de conteúdo: (i) categorização - operação de classificação de elementos constitutivos de um conjunto por diferenciação; e (ii) reagrupamento segundo o gênero (analogia), a partir de categorias gerais (rubricas ou classes), as quais reúnem um grupo de elementos ou unidades de registro sob um título genérico (BARDIN, 20I I). Dessa forma, ao final, todos os programas evidenciados nos relatórios GRI de 2015 e 2016 da amostra foram agrupados em categorias e subcategorias desenvolvidas no estudo, a partir de uma avaliação por pares.

A segunda etapa consistiu na análise de correlação entre os atributos organizacionais e os programas de capacitação da amostra. Conforme apresentado no tópico anterior, foram delineadas duas hipóteses principais, que se desdobram em sete hipóteses operacionais relacionadas aos atributos institucionais $(\mathrm{HIa}, \mathrm{HIb}, \mathrm{HIc}, \mathrm{HId}$, $\mathrm{Hle}, \mathrm{HIf}, \mathrm{HIg}$ ) e sete hipóteses operacionais relacionadas aos atributos humanos $(\mathrm{H} 2 \mathrm{a}, \mathrm{H} 2 \mathrm{~b}, \mathrm{H} 2 \mathrm{c}, \mathrm{H} 2 \mathrm{~d}, \mathrm{H} 2 \mathrm{e}, \mathrm{H} 2 \mathrm{f}, \mathrm{H} 2 \mathrm{~g})$. Para verificação das referidas hipóteses, procedeu-se à realização de testes de correlação entre as variáveis.

A correlação entre variáveis é frequentemente um dos propósitos das pesquisas empíricas, tendo em vista que sua possível existência orienta análises e conclusões, assim como evidencia achados de investigação. Em estudos sobre a mesma temática, o emprego da técnica também foi realizado por outros pesquisadores (HAHN; KÜHNEN, 2013; EHNERT et al., 20I5; GUERCl et al., 20 I8), baseando-se em lacunas existentes.

QUADRO 4 - Atributos humanos e respectiva operacionalização

\begin{tabular}{|c|c|}
\hline Atributos & Operacionalização \\
\hline Número de conselheiros & Número médio anual de membros do conselho de administração \\
\hline Formação do chairman & Formação do presidente do conselho de administração \\
\hline HRM expertise dos conselheiros & Experiência ou vivência prática em áreas de desenvolvimento humano \\
\hline Independência do chairman & $\begin{array}{l}\text { Variável dummy, que informa se há independência entre os cargos do presidente do } \\
\text { conselho de administração e da diretoria executiva }\end{array}$ \\
\hline Número de diretores & Número médio anual de membros da diretoria executiva \\
\hline Formação do CEO & Formação do presidente da diretoria executiva \\
\hline HRM expertise dos diretores & Experiência ou vivência prática em áreas de desenvolvimento humano \\
\hline
\end{tabular}

Fonte: Elaborado pelos autores. 
Considerando-se a amplitude amostral $(n=104)$, o relato dos programas de capacitação e os atributos organizacionais - institucionais (Quadro I) e humanos (Quadro 2) - da amostra, recorreu-se ao emprego da correlação de Pearson, visto o comportamento próximo da normalidade das variáveis estudadas, a partir da aplicação do Teorema do Limite Central. Para tanto, na análise geral, foi considerado o número total de programas evidenciados por cada empresa no biênio em análise (20I5-20l6).

Por fim, de posse da categorização dos programas, após a realização das análises de correlação de Pearson entre cada um dos atributos organizacionais e a divulgação de programas de maneira geral, visando testar as hipóteses operacionais da pesquisa, foram aplicados testes de correlação entre cada um dos atributos institucionais e humanos e as categorias dos programas.

\section{RESULTADOS \\ Categorização dos programas de capacitação}

A categorização dos programas das maiores companhias abertas do Brasil é vista como contribuição basilar deste estudo que tem foco no reporting de pessoas em sustentabilidade. Inicialmente, na análise de conteúdo, foram definidas categorias gerais elencadas pela comunidade científica no tocante à gestão do capital humano. Em seguida, foi investigada nos relatórios de sustentabilidade a aplicabilidade do quadro preliminar, fazendo-se os ajustes necessários, ao se identificar programas que não se relacionavam a nenhuma das categorias inicialmente definidas.

Ao todo, investigou-se e adaptou-se o quadro geral de categorização dos programas de capacitação no tocante aos 104 rela- tórios analisados, de modo a não se excluir nenhum programa. $O$ Quadro 5 apresenta as áreas de capacitação e suas respectivas categorias e subcategorias, seguidas dos aspectos-chave considerados para agrupamento durante a análise de conteúdo.

A categorização realizada torna possível a identificação da proporção de programas das empresas em cada área e suas respectivas categorias e subcategorias, assim como a descoberta de similaridades e divergências dos escopos dos programas relatados no biênio 2015-2016.

Após a categorização dos programas, considerando-se os aspectos-chave para agrupamento, procedeu-se à mensuração do número de programas evidenciados com base nessa categorização. Para ilustrar o perfil dos programas de capacitação evidenciados nos 104 relatórios da amostra, elaborou-se a estatística descritiva das suas categorias e subcategorias, por área de capacitação (Tabela 2).

A partir das informações da Tabela 2, levantadas com base na categorização dos programas (Quadro 5), nota-se certa heterogeneidade. Resultado semelhante nas companhias peruanas foi obtido por Pozas e Jauregui (20I2). Na amostra aqui estudada, a categoria Responsabilidade socioambiental, vinculada à área de Desenvolvimento social corporativo, reuniu a maior média $(8,68)$ de programas relatados no biênio sob análise, especialmente vinculados às subcategorias: Saúde e segurança $(4,25)$, e, Consciência social e ambiental $(3,35)$. Os programas dessa categoria foram encontrados em 103 dos 104 relatórios analisados, o que evidencia a visibilidade dada a essas práticas nas maiores companhias abertas brasileiras. Esse achado está alinhado com a crescente valorização desses aspectos 
QUADRO 5 - Áreas, categorias, subcategorias e aspectos-chave relativos aos programas de capacitação identificados

\begin{tabular}{|c|c|c|}
\hline \multicolumn{3}{|c|}{ ÁREA - DESENVOLVIMENTO PROFISSIONAL } \\
\hline Categoria & Subcategoria & Aspecto-chave \\
\hline \multirow{3}{*}{ 1. Jovens talentos } & Jovem aprendiz & $\begin{array}{l}\text { Atração e desenvolvimento de jovens inscritos em programa de aprendiza- } \\
\text { gem, formação técnico-profissional metódica, compatível com o seu desen- } \\
\text { volvimento }\end{array}$ \\
\hline & Estágio & Atração e desenvolvimento de graduandos (universitários) \\
\hline & Trainee & $\begin{array}{l}\text { Atração e desenvolvimento de recém-formados ou destaques em master of } \\
\text { business administration (MBA) }\end{array}$ \\
\hline \multirow{4}{*}{$\begin{array}{l}\text { 2. Educação } \\
\text { continuada }\end{array}$} & Mobilidade interna & $\begin{array}{l}\text { Mobilidade regional, nacional ou internacional, transferências e outras oportu- } \\
\text { nidades profissionais na empresa, ou banco de talentos. }\end{array}$ \\
\hline & Formação educacional & $\begin{array}{l}\text { Formação educacional básica, técnica, profissionalizante, graduação e pós- } \\
\text { graduação, intercâmbio acadêmico ou estudo de línguas estrangeiras. }\end{array}$ \\
\hline & $\begin{array}{l}\text { Reabilitação / recolocação / } \\
\text { requalificação }\end{array}$ & $\begin{array}{l}\text { Reabilitação de empregados acidentados, apoio para recolocação ou requali- } \\
\text { ficação profissional. }\end{array}$ \\
\hline & $\begin{array}{l}\text { Preparo para aposenta- } \\
\text { doria }\end{array}$ & Planejamento e recursos preparatórios para aposentadoria ou demissão. \\
\hline \multicolumn{3}{|c|}{ ÁREA - DESENVOLVIMENTO HUMANO } \\
\hline Categoria & Subcategoria & Aspecto-chave \\
\hline \multirow{3}{*}{ 3. Comportamental } & Liderança & Formação de (novos) líderes \\
\hline & $\begin{array}{l}\text { Coaching e } \\
\text { mentoring }\end{array}$ & $\begin{array}{l}\text { Ferramentas comportamentais, feedback } 360 \text { graus, mapa de competências, } \\
\text { guia, orientação, transferência de insights. }\end{array}$ \\
\hline & Finanças pessoais & Educação financeira e orçamento familiar. \\
\hline \multirow{3}{*}{ 4. Técnico } & Habilidades inovadoras & Inovação de plataformas e sistemas operacionais. \\
\hline & Habilidades de gestão & Educação continuada para gestores. \\
\hline & Habilidades operacionais & $\begin{array}{l}\text { Qualidade dos processos, atividades específicas de vendas, de compras, de } \\
\text { marketing etc. }\end{array}$ \\
\hline \multicolumn{3}{|c|}{ ÁREA - DESENVOLVIMENTO SOCIAL CORPORATIVO } \\
\hline Categoria & Subcategoria & Aspecto-chave \\
\hline \multirow[b]{2}{*}{ 5. Integridade } & $\begin{array}{l}\text { Compliance, ética e } \\
\text { anticorrupção }\end{array}$ & $\begin{array}{l}\text { Conformidade, ética, integridade, anticorrupção, gestão de riscos e segurança } \\
\text { da informação. }\end{array}$ \\
\hline & Cultura corporativa & $\begin{array}{l}\text { Comunicação interna, canais de relacionamento, clima laboral; organização } \\
\text { de eventos ou ações de integração nacionais e internacionais; e apoio à par- } \\
\text { ticipação de colaboradores em eventos externos, universidade ou academia } \\
\text { corporativa. }\end{array}$ \\
\hline \multirow{3}{*}{$\begin{array}{l}\text { 6. Responsabilidade } \\
\text { socioambiental }\end{array}$} & Saúde e segurança & $\begin{array}{l}\text { Saúde ocupacional, prevenção e ergonomia, segurança de processo e segu- } \\
\text { rança ocupacional. }\end{array}$ \\
\hline & $\begin{array}{l}\text { Consciência social e } \\
\text { ambiental }\end{array}$ & Consciência social, consciência ambiental, voluntariado ou instrutória. \\
\hline & $\begin{array}{l}\text { Diversidade, equidade e } \\
\text { inclusão }\end{array}$ & $\begin{array}{l}\text { Respeito, equidade e inclusão entre raças, gêneros, idades e tipos de neces- } \\
\text { sidades especiais. }\end{array}$ \\
\hline
\end{tabular}

Fonte: Elaborado pelos autores. 
TABELA 2 - Estatística descritiva dos programas de capacitação das empresas, por categoria e subcategoria

\begin{tabular}{|c|c|c|c|c|}
\hline ÁREA 1 - Desenvolvimento Profissional & Média & Desvio-padrão & Mínimo & Máximo \\
\hline Categoria 1. Jovens talentos & 1,67 & 1,651 & 0 & 6 \\
\hline Jovem aprendiz & 0,50 & 0,557 & 0 & 2 \\
\hline Estágio & 0,58 & 0,720 & 0 & 3 \\
\hline Trainee & 0,60 & 0,887 & 0 & 4 \\
\hline Categoria 2. Educação continuada & 2,85 & 2,392 & 0 & 11 \\
\hline Mobilidade interna & 0,36 & 0,606 & 0 & 3 \\
\hline Formação educacional & 1,88 & 1,939 & 0 & 9 \\
\hline Reabilitação/recolocação/requalificação & 0,17 & 0,511 & 0 & 4 \\
\hline Preparo para aposentadoria & 0,44 & 0,680 & 0 & 2 \\
\hline ÁREA 2 - Desenvolvimento Humano & Média & Desvio-padrão & Mínimo & Máximo \\
\hline Categoria 3. Comportamental & 2,50 & 2,302 & 0 & 11 \\
\hline Liderança & 1,01 & 1,219 & 0 & 8 \\
\hline Coaching e mentoring & 1,18 & 1,252 & 0 & 5 \\
\hline Finanças pessoais & 0,31 & 0,976 & 0 & 7 \\
\hline Categoria 4. Técnico & 2,36 & 2,076 & 0 & 10 \\
\hline Habilidades inovadoras & 1,05 & 1,280 & 0 & 8 \\
\hline Habilidades de gestão & 0,45 & 0,736 & 0 & 3 \\
\hline Habilidades operacionais & 0,86 & 1,226 & 0 & 6 \\
\hline ÁREA 3 - Desenvolvimento Social Corporativo & Média & Desvio-padrão & Mínimo & Máximo \\
\hline Categoria 5. Integridade & 3,63 & 1,952 & 1 & 9 \\
\hline Compliance, ética e anticorrupção & 1,08 & 0,942 & 0 & 4 \\
\hline Cultura corporativa & 2,55 & 1,695 & 0 & 7 \\
\hline Categoria 6. Responsabilidade socioambiental & 8,68 & 4,668 & 0 & 23 \\
\hline Saúde e segurança & 4,25 & 3,300 & 0 & 17 \\
\hline Consciência social e ambiental & 3,35 & 2,267 & 0 & 13 \\
\hline Diversidade, equidade e inclusão & 1,09 & 1,301 & 0 & 8 \\
\hline
\end{tabular}

Fonte: Elaborada pelos autores.

na sociedade contemporânea (EHNERT; HARRY; ZINK, 20I4; EHNERT et al., 20I5; GUERCI et al., 20I8; LELEBINA; GAND, 2018) e com a legislação vigente no país, bem como por ser uma das principais informações que se espera encontrar em um relato de sustentabilidade.

Além da categoria Responsabilidade socioambiental, destaca-se a categoria Integridade, com uma média de 3,63 programas nas empresas. Bortoluzzi, Genari e Macke (2017) identificaram que a aprendizagem formal, baseada no ensino superior, é a principal forma de aquisição do capital humano; por sua vez, a Tabela 2 indica que a categoria Educação continuada teve apenas a terceira melhor média $(2,85)$ entre os programas. A categoria Jovens talentos apresentou o menor número de programas, com média inferior a 2 , sendo a categoria da área Desenvolvimento profissional. Tal fato indica menor investimento em programas ofertados a jovens aprendizes, estagiários e trainees, apesar das pressões externas, bem como de incentivos do governo para o desenvolvimento de programas voltados para esses públicos específicos. Cabe mencionar que a $\mathrm{TCH}$ aponta como risco o investimento na formação de pessoas jovens, principalmente as que 
fazem uso de mobilidade externa para qualificar, tendo em vista que os jovens podem decidir não retornar (KAROLCZAK; SOUZA, 20 I7; SCHULTZ, I96I).

Após a categorização dos programas reportados pelas empresas nos seus relatórios, procedeu-se à análise de correlação entre os atributos organizacionais e o reporte dos programas.

\section{Atributos correlacionados com o relato de programas de capacitação}

A Tabela 3 apresenta a correlação entre os atributos organizacionais e o reporting de programas nas empresas, de uma forma geral, conforme número total de programas relatados no biênio 2015-2016.

$\mathrm{Na}$ Tabela 3, verifica-se que dos 14 atributos considerados no estudo, apenas três estão estatisticamente correlacionados com o relato de programas. $O$ porte e a internacionalização apresentaram correlação positiva com o reporting de programas, corroborando Almeida e Aterido
(20I5) e Félix Júnior et al. (20I8). Quanto à internacionalização, nota-se o maior grau de exigência do mercado externo no que concerne a políticas de gestão do capital humano, convergente com os estudos de Rosolen e Maclennam (2016) e Petry et al. (20I4). Destarte, são confirmadas as hipóteses $\mathrm{Hla}$ e HIg.

Quanto aos atributos humanos, apenas o número de diretores apresentou correlação positiva com o relato de programas, indicando que quanto maior for o número de diretores da empresa, maior será o número de programas de capacitação ali relatados. Esse achado não confirma a hipótese $\mathrm{H} 2 \mathrm{c}$, e vai de encontro ao estudo de Cortés et al. (2017), que indica uma correlação negativa entre $\circ$ tamanho da diretoria $e$ o desempenho econômico, e sugere que, quanto maior for o número de diretores, mais lento será o processo decisório, tornando-o ineficiente em suas funções. Tal resultado, contudo, pode indicar um ponto de partida para novas pesquisas.

TABELA 3 - Atributos organizacionais e sua correlação com o relato de programas de capacitação

\begin{tabular}{|c|c|c|c|}
\hline \multicolumn{2}{|r|}{ Atributos } & Correlação de Pearson & Significância \\
\hline \multirow{7}{*}{ 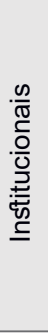 } & Porte & $0,339^{(* *)}$ & 0,000 \\
\hline & Rentabilidade & 0,113 & 0,253 \\
\hline & Endividamento & 0,132 & 0,181 \\
\hline & Setor & $-0,161$ & 0,103 \\
\hline & Idade & 0,107 & 0,278 \\
\hline & Governança corporativa & $-0,128$ & 0,196 \\
\hline & Internacionalização & $0,233^{(*)}$ & 0,017 \\
\hline \multirow{7}{*}{ 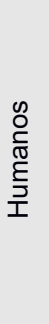 } & Número de conselheiros & $-0,105$ & 0,288 \\
\hline & Formação do chairman & 0,022 & 0,828 \\
\hline & HRM expertise dos conselheiros & 0,022 & 0,822 \\
\hline & Independência do chairman & $-0,040$ & 0,686 \\
\hline & Número de diretores & $0,226^{(*)}$ & 0,021 \\
\hline & Formação do CEO & $-0,121$ & 0,219 \\
\hline & HRM expertise dos diretores & 0,135 & 0,171 \\
\hline
\end{tabular}

$\left(^{*}\right)$ significante a $5 \% ;\left(^{* *}\right)$ significante a $10 \%$.

Fonte: Elaborada pelos autores. 
Assim, apesar de as duas hipóteses principais $(\mathrm{HI}$ e $\mathrm{H2}$ ) terem sido rejeitadas, seguindo a sugestão de Gaspar et al. (2016) de que as práticas de gestão do capital humano devem ser analisadas de forma geral e agrupadas, na subseção seguinte explicitam-se os resultados da correlação entre os atributos organizacionais e as categorias dos programas de capacitação (Quadro 5).

\section{Atributos correlacionados ao relato dos programas de capacitação, por categoria}

Para a análise de correlação entre os atributos organizacionais e a divulgação de programas por categoria, foram aplicados seis testes de correlação de Pearson. A Tabela 4 exibe uma síntese dos atributos organizacionais que apresentaram correlação significante com o reporting de programas, por categoria.

Os programas de capacitação da categoria Jovens talentos estão presentes em 75\% da amostra, o que já se esperava, tendo em vista a regulamentação brasileira, a saber: Lei n. 10.097/2000 e Lei n. I I. I80/2005, que regulamentam os programas de jovem aprendiz; Lei n. I I.788/2008, que regulamenta o estágio; e a Consolidação das Leis do Trabalho - CLT, Decreto-lei n. 5.452/1943, que regulamenta o trainee). Ainda quanto à categoria Jovens talentos, nota-se que os programas relatados se mostraram estatisticamente correlacionados: negativamente com o setor e com a formação do CEO; e, positivamente com o endividamento. Dessa forma, todas as correlações significantes (Tabela 4) apresentam sentidos opostos ao do relacio-

TABELA 4 - Atributos organizacionais correlacionados com o relato de programas de capacitação, por categoria

\begin{tabular}{|c|c|c|}
\hline Atributos correlacionados & Correlação de Pearson & Significância \\
\hline \multicolumn{3}{|l|}{ Categoria 1. Jovens talentos } \\
\hline Setor & $-0,326^{(* *)}$ & 0,001 \\
\hline Endividamento & $0,226^{(*)}$ & 0,21 \\
\hline Formação do CEO & $-0,267^{(* *)}$ & 0,006 \\
\hline \multicolumn{3}{|l|}{ Categoria 2. Educação continuada } \\
\hline Porte & $0,227^{(*)}$ & 0,20 \\
\hline Governança corporativa & $-0,319^{(* *)}$ & 0,001 \\
\hline Internacionalização & $0,272^{(* *)}$ & 0,005 \\
\hline Número de diretores & $0,250^{(*)}$ & 0,011 \\
\hline HRM expertise dos diretores & $0,323^{(* *)}$ & 0,001 \\
\hline \multicolumn{3}{|l|}{ Categoria 3. Comportamental } \\
\hline Porte & $0,256^{(* *)}$ & 0,009 \\
\hline Rentabilidade & $0,243^{(*)}$ & 0,013 \\
\hline Número de diretores & $0,329^{(* *)}$ & 0,001 \\
\hline \multicolumn{3}{|l|}{ Categoria 5. Integridade } \\
\hline Porte & $0,255^{(*)}$ & 0,009 \\
\hline Independência do chairman & $-0,283^{(* *)}$ & 0,004 \\
\hline Número de diretores & $0,227^{(*)}$ & 0,020 \\
\hline \multicolumn{3}{|c|}{ Categoria 6. Responsabilidade socioambiental } \\
\hline Porte & $0,207^{(*)}$ & 0,035 \\
\hline
\end{tabular}

$\left({ }^{*}\right)$ significante a $5 \% ;\left({ }^{* *}\right)$ significante a $10 \%$.

Fonte: Elaborada pelos autores. 
namento esperado entre esses atributos e o reporting de programas de capacitação.

Quanto à Educação continuada, três atributos institucionais (porte, governança corporativa e internacionalização) e dois atributos humanos (número de diretores e HRM expertise dos diretores) mostraram correlações significativas com o relato de programas. Note-se que quanto mais HRM expertise dos diretores mais as empresas relatam programas de capacitação da categoria Educação continuada, corroborando Carrasco (20I I), Díez-De-Castro, Díez-Martín e Vázquez-Sánche (20I5). Percebe-se que das correlações significantes identificadas, além da governança corporativa, o número de diretores também aponta sentido oposto ao relacionamento esperado com o relato de programas.

Os programas da categoria Comportamental relatados pela amostra mostram-se positivamente correlacionados com o porte e a rentabilidade, ratificando os achados de Utrilla et al. (20I5), e com o número de diretores.

Como a categoria Técnico, que reúne três subcategorias (Habilidades inovadoras, Habilidades de gestão e Habilidades operacionais), não apresentou correlação significante com nenhum dos atributos considerados no estudo, a mesma não foi disposta na Tabela 4. Torna-se necessário esclarecer que essa categoria está relacionada a programas mais específicos nas áreas de inovação e processos de melhoria.

Ademais, todas as empresas da amostra relatam em seus relatórios programas da categoria Integridade, visto que os investimentos em ética e compliance permeiam entre as recomendações das melhores práticas de governança. $\mathrm{Na}$ Tabela 4, identifica-se uma correlação significante e positiva entre o relato de programas dessa categoria e o porte e o número de diretores. No en- tanto, nota-se uma correlação significante e negativa entre o relato e a independência do chairman, o que vai de encontro a Mascena et al. (2016).

Ainda com base na Tabela 4, verifica-se apenas uma correlação significante e positiva entre o porte e o relato de programas da categoria Responsabilidade socioambiental, que registra o maior número de programas evidenciados pelas empresas da amostra no biênio.

\section{Síntese dos achados}

O Quadro 6 mostra a síntese dos achados da pesquisa, considerando-se os resultados esperados com base no suporte teórico e as hipóteses levantadas, de forma geral e específica.

Observa-se que apesar de apenas três atributos organizacionais - porte, internacionalização e número de diretores - apresentarem correlação significante com o relato de programas de capacitação, considerando-se o número de programas em geral, - resultado esperado, entre os atributos e o relato de programas de capacitação, confirmou-se em 9 dos 14 atributos analisados, apesar da ausência de significância em 6 deles. Cabe ressaltar que em 5 do total de atributos organizacionais, conforme destaque em vermelho, notou-se que o resultado (sinal) observado difere do esperado, este último constituído a partir das recomendações da literatura. Esse achado apontou para a relevância de uma análise pormenorizada ou específica, que foi, portanto, desenvolvida no estudo.

Analisando-se, de forma específica, os achados das correlações vinculadas aos atributos institucionais, quanto à hipótese $\mathrm{HIa}$, verifica-se que o porte se apresentou significante e positivamente correlaciona- 
QUADRO 6 - Síntese das correlações entre os potenciais atributos e o relato de programas de capacitação

\begin{tabular}{|c|c|c|c|c|c|c|c|c|c|}
\hline \multirow{2}{*}{\multicolumn{2}{|c|}{ Atributos }} & \multirow{3}{*}{$\begin{array}{c}\text { Resultado } \\
\text { Esperado } \\
+ \\
\end{array}$} & \multicolumn{7}{|c|}{ Resultado Observado } \\
\hline & & & Geral & Categ. 1 & Categ. 2 & Categ. 3 & Categ. 4 & Categ. 5 & Categ. 6 \\
\hline \multirow{7}{*}{ 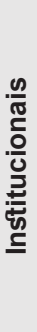 } & Porte & & $+(* *)$ & - (NS) & $+^{(* *)}$ & $+(* *)$ & $+(\mathrm{NS})$ & $+^{(* *)}$ & $+^{(*)}$ \\
\hline & Rentabilidade & + & $+(\mathrm{NS})$ & $+(\mathrm{NS})$ & $+(\mathrm{NS})$ & $+^{(*)}$ & - (NS) & $+(\mathrm{NS})$ & - (NS) \\
\hline & Endividamento & - & $+(\mathrm{NS})$ & $+^{(*)}$ & $+(\mathrm{NS})$ & $+(\mathrm{NS})$ & - (NS) & $+(\mathrm{NS})$ & $+(\mathrm{NS})$ \\
\hline & Setor & + & - (NS) & $-(* *)$ & - (NS) & - (NS) & $+(\mathrm{NS})$ & - (NS) & - (NS) \\
\hline & Idade & + & $+(\mathrm{NS})$ & - (NS) & $+(\mathrm{NS})$ & $+(\mathrm{NS})$ & - (NS) & $+(\mathrm{NS})$ & $+(\mathrm{NS})$ \\
\hline & Governança corporativa & + & - (NS) & $-(N S)$ & $-(* *)$ & - (NS) & $-(N S)$ & $-(N S)$ & $+(\mathrm{NS})$ \\
\hline & Internacionalização & + & $+^{(*)}$ & $+(\mathrm{NS})$ & $+^{(* *)}$ & $+(\mathrm{NS})$ & $+(\mathrm{NS})$ & $+(\mathrm{NS})$ & $+(\mathrm{NS})$ \\
\hline \multirow{7}{*}{ 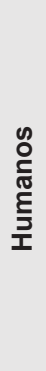 } & Número de conselheiros & - & - (NS) & - (NS) & - (NS) & - (NS) & - (NS) & - (NS) & $-(N S)$ \\
\hline & Formação do chairman & + & $+(\mathrm{NS})$ & - (NS) & $+(\mathrm{NS})$ & - (NS) & $+(\mathrm{NS})$ & $+(\mathrm{NS})$ & $+(\mathrm{NS})$ \\
\hline & $\begin{array}{l}\text { HRM expertise dos } \\
\text { conselheiros }\end{array}$ & + & $+(\mathrm{NS})$ & - (NS) & - (NS) & - (NS) & - (NS) & - (NS) & $+(\mathrm{NS})$ \\
\hline & Independência do chairman & + & - (NS) & $+(\mathrm{NS})$ & $+(\mathrm{NS})$ & - (NS) & $+(\mathrm{NS})$ & $-(* *)$ & $+(\mathrm{NS})$ \\
\hline & Número de diretores & - & $+^{(*)}$ & - (NS) & $+^{(*)}$ & $+(* *)$ & - (NS) & $+(\mathrm{NS})$ & $+(N S)$ \\
\hline & Formação do CEO & + & - (NS) & $-(* *)$ & $+(\mathrm{NS})$ & - (NS) & - (NS) & - (NS) & - (NS) \\
\hline & HRM expertise dos diretores & + & $+(\mathrm{NS})$ & $+(\mathrm{NS})$ & $+^{(* *)}$ & $+(N S)$ & $+(\mathrm{NS})$ & - (NS) & $+(N S)$ \\
\hline
\end{tabular}

$\left(^{*}\right)$ significante a 5\%; $\left.{ }^{* *}\right)$ significante a 10\%; (NS) correlação não significante; (+) correlação positiva; (-) correlação negativa.

Fonte: Elaborado pelos autores.

do com as categorias 2, 3, 5 e 6, corroborando Almeida e Aterido (2015) quanto à correlação positiva entre investimento em treinamento profissional e o porte nas firmas de 99 países. Quanto à hipótese HIb, notou-se que a rentabilidade apresenta correlação significante e positiva especificamente com a categoria 3, corroborando Utrilla e Grande-Torraleja (2012), na medida em que estes identificaram que 0 desempenho das empresas é resultado de investimento no capital humano. No tocante à hipótese $\mathrm{HIc}$, o endividamento se mostrou significante e positivamente correlacionado especificamente com a categoria I, refutando Mamede et al. (20I7) segundo os quais o endividamento é inversamente proporcional ao porte da empresa. Quanto à hipótese $\mathrm{HId}$, o setor se apresentou significante e negativamente correlacionado especificamente com a categoria I, indo de encontro à tese de Gaspar et al. (2016) - cabe destacar que o sinal da correlação identificada considerou a variável categórica que agregou os 16 setores da base Economática, sendo os maiores números atribuídos aos setores mais inovativos. Quanto à hipótese $\mathrm{Hle}$, não se observou correlação significante específica de nenhuma categoria com a idade da empresa. Sobre a hipótese HIf, a governança mostrou-se significante e negativamente correlacionada especificamente com a categoria 2, refutando Beuren e Marcello (20I6), segundo os quais o capital humano é o recurso mais importante para as empresas com boa governança. Quanto à hipótese $\mathrm{HI} \mathrm{g}$, a internacionalização mostrou-se significante e positivamente correlacionada com a categoria 2, refutando Rosolen e Maclennam (2016), que não identificaram influência da internacionalização no gerenciamento de recursos humanos e na responsabilidade social.

Considerando-se, de forma específica, os achados das correlações vinculadas aos atributos humanos, quanto às hipóteses $\mathrm{H} 2 \mathrm{a}, \mathrm{H} 2 \mathrm{~b}$ 
e $\mathrm{H} 2 c$, o número de conselheiros, a formação do chairman e a HRM expertise dos conselheiros não apresentaram correlação significante específica com nenhuma categoria de programa. Quanto à hipótese $\mathrm{H} 2 \mathrm{~d}$, a independência do chairman se apresentou significante e negativamente correlacionada especificamente com a categoria 5, refutando Mascena et al. (2016). Quanto à hipótese H2e, o número de diretores mostrou-se significante e positivamente correlacionado com as categorias $2 \mathrm{e}$ 3 , refutando Cortés et al. (20I7), segundo os quais, quanto maior for a diretoria executiva, mais lento é o processo decisório. Quanto à hipótese $\mathrm{H} 2 \mathrm{f}$, a formação do CEO apresentouse significante e negativamente correlacionada apenas com a categoria I, refutando os achados de Carrasco (20I I). Por fim, no que se refere à hipótese $\mathrm{H} 2 \mathrm{~g}$, a $H R M$ expertise dos diretores mostrou-se positivamente correlacionada especificamente com a categoria 2 , corroborando Díez-De-Castro, Díez-Martín eVázquez-Sánchez (20I5).

\section{CONCLUSÃO}

O relato de programas de capacitação configura-se como $\circ$ reporting de investimento em capital humano que tem se tornado o grande diferencial da organização, dada a dificuldade de ser copiado, imitado ou adaptado, podendo ser fonte de vantagem competitiva. Assim, investigou-se os programas de capacitação relatados pelas maiores companhias abertas brasileiras, identificando suas categorias e os atributos institucionais e humanos possivelmente correlacionados com o seu relato. Contudo, conclui-se que é incipiente à correlação entre o reporting de programas de capacitação e os atributos organizacionais investigados, sendo mais evidente quando os programas são tratados por categoria.
Sem dúvida, a principal contribuição do estudo reside na categorização dos programas de capacitação evidenciados pelas maiores companhias abertas no Brasil. A análise de conteúdo dos relatórios GRI de 2015 e 2016 proporcionou a identificação do perfil dos programas relatados pelas empresas, sendo possível identificar que eles estão contemplados em três grandes áreas (Desenvolvimento Profissional, Desenvolvimento Humano e Desenvolvimento Social Corporativo), que possuem seis categorias (Jovens talentos, Educação continuada, Comportamental, Técnico, Integridade e Responsabilidade socioambiental).

No geral, a análise de conteúdo possibilitou a identificação de 2.255 programas de capacitação relatados pelas empresas. Em síntese, $40 \%$ do total de programas pertencem à Responsabilidade socioambiental, seguida por: Integridade (I7\%), Educação continuada (I3\%), Comportamental (II\%), Técnico ( $11 \%$ ) e Jovens talentos (8\%). Ademais, há de se destacar a concentração do reporting de programas de Desenvolvimento social corporativo (57\%). Tal resultado vai ao encontro de Schultz (I96I), responsável pela formulação inicial da $\mathrm{TCH}$, que indica que dentre as atividades que podem meIhorar a capacidade humana destacam-se: facilidades em serviços de saúde, para garantir uma expectativa de vida com vigor e vitalidade; e educação formalizada nos três níveis, desde as séries iniciais até o superior.

Destacam-se dentre as limitações do estudo o tamanho e o perfil da amostra, inviabilizando que seus resultados sejam extrapolados para outros tipos de empresa, bem como a delimitação temporal (2015 e 2016). Não se pretende, de forma alguma, exaurir os conhecimentos sobre esse fenômeno a partir desta pesquisa. Nesse 
sentido, sugere-se o desenvolvimento de pesquisas sobre a relação aqui investigada com a ampliação da amostra e do período de análise, bem como a aplicação de técnicas estatísticas mais robustas. Além disso, recomenda-se a realização futura de estudos qualitativos que se debrucem sobre a categorização dos programas de capacitação e sobre a questão do isomorfismo institucional entre as ações na área de gestão de pessoas.

Apesar das limitações apontadas, o estudo oferece contribuições importantes. De forma prática, proporciona contribuição acadêmica, a partir da categorização do reporting de programas de capacitação no Bra- sil e a identificação de alguns atributos organizacionais correlacionados, o que pode ser aprofundado em futuras pesquisas.

Como contribuição gerencial, apresenta um mapeamento das principais práticas sustentáveis de gestão do capital humano, servindo de ponto de partida para empresas que têm a intenção de iniciar a sua institucionalização. Ademais, como contribuição socioeconômica, apresenta características institucionais relevantes no contexto nacional, e gera indícios explicativos para o reporting de pessoas em sustentabilidade, chamando a atenção para esse importante tema para o contexto empresarial e econômico do país. 


\section{REFERÊNCIAS}

ALMEIDA, R.; ATERIDO, R. Investing in formal on-the-job training: are SMEs lagging much behind? IZA Journal of Labor \& Development, v. 4, n. I, p. I-23, 2015.

AMBROZINI, L. Pressões internas e externas na utilização de padrões de divulgação de informações socioambientais amplamente aceitos: uma análise sobre estrutura de governança corporativa, ambiente institucional e a perspectiva de legitimação. Revista Contemporânea de Contabilidade, v. I4, n. 3I, p. 03-25, 2017. ASSUNÇÃO, R. M.; DE LUCA, M. M. M.; VASCONCELOS, A. C. Complexity and corporate governance: an analysis of companies listed on the BM\&FBovespa. Revista Contabilidade \& Finanças, v. 28, n. 74, p. 2I3-228, 2017.

BARDIN, L. Análise de conteúdo. São Paulo: Edições 70, 201 I.

BEUREN, I. M.; MARCELLO, I. E. Relação da importância dos recursos estratégicos com as medidas de desempenho em empresas brasileiras. Revista Ibero-Americana de Estratégia, v. I5, n. I, P. 65-82, 2016.

BORTOLUZZI, F. R.; GENARI, D.; MACKE, J.A obsolescência do capital humano nas empresas de tecnologia da informação: um estudo na Associação Internetsul. Revista Alcance, v. 24, n. 4, p. 5|3-534, 2017.

BRASIL. Decreto-lei n. 5.452, de $I^{\circ}$ de maio de 1943. Aprova a Consolidação das Leis do Trabalho. Brasília. 1943. Disponível em: <http://www.planalto.gov.br/ ccivil_03/Decreto-Lei/Del5452. htm>. Acesso em: 30 out. 2019.

BRASIL. Lei n. 10.097, de 19 de dezembro de 2000. Altera dispositivos da Consolidação das Leis do Trabalho - CLT, aprovada pelo
Decreto-Lei no 5.452, de lo de maio de 1943. Brasília. 2000. Disponível em: <http://www.planalto.gov.br/ccivil_03/LEIS/LI0097. htm>. Acesso em: 30 out. 2019.

BRASIL. Lei n. I I. I 80, de 23 de setembro de 2005. Institui o Projeto Escola de Fábrica, autoriza a concessão de bolsas de permanência a estudantes beneficiários do Programa Universidade para Todos - PROUNI, institui o Programa de Educação Tutorial - PET, e dá outras providências. Brasília. 2005. Disponível em: <http:// www.planalto.gov.br/ccivil_03/ Ato2004-2006/2005/Lei/L I I I80. htm>. Acesso em: 12 set. 2019.

BRASIL. Lei n. I I.788, de 25 de setembro de 2008. Altera a legislação que regulamenta legislação nacional alusiva ao contrato de estágio para estudantes do ensino regular. Diário Oficial da União. Brasília. 2008. Disponível em: <http://www.planalto.gov.br/ ccivil_03/_Ato2007-2010/2008/ Lei/LI I788.htm>. Acesso em: 10 out. 2019.

CAMPOS, A. L.; SANTOS, N. M. B. F.; RODRIGUES, J. L. K. Gestão de recursos humanos e avaliação de desempenho por competências: análise do processo em uma empresa do Vale do Paraíba. ENIAC Pesquisa, v. I, n. 3, p. 33-53, 20 I4.

CARRASCO, C. La economía del cuidado: planteamiento actual y desafíos pendientes. Revista de Economía Crítica, v. I I, p. 205225,20 I I.

CORTÉS, D. L. et al. Tamaño e independencia de la junta directiva y su relación con el desempeño económico: un análisis para empresas familiares y no familiares. AD-minister, v. 3I, p. 5-23, 2017. DÍEZ-DE-CASTRO, E.; DÍEZ-MARTÍN, F; VÁZQUEZ-SÁNCHEZ,
A. Antecedentes de la institucionalización de las organizaciones. Cuadernos de Gestión, v. I5, n. I, P. I5-38, 20 I 5.

EHNERT, I.; HARRY, W. Recent developments and future prospects on sustainable human resource management: Introduction to the special issue. Management Revue, v. 23, p. 22 I-238, 2012.

EHNERT, I.; HARRY, W.; ZINK, K. J. Sustainability and HRM: An introduction to the field. In: EHNERT, I.; HARRY,W.; ZINK, K.J. (Ed.), Sustainability and human resource management: Developing sustainable business organizations (pp. 3-32). Heidelberg: Springer, 2014.

EHNERT, I. et al. Reporting on sustainability and HRM: a comparative study of sustainability reporting practices by the world's largest companies. The International Journal of Human Resource Management, v. 27 , n. I, p. 88$108,2015$.

ENGELEN, A. et al. Entrepreneurial orientation, firm performance, and the moderating role of transformational leadership behaviors. Journal of Management, v. 4I, n. 4, p. 1069-1097, 2015.

FÉLIX JÚNIOR, L.A. et al. Divulgação de informações sobre recursos humanos e os fatores que influenciam sua evidenciação voluntária nos relatórios da administração. Enfoque: Reflexão Contábil, v. 37, n. I, p. I29-|46, 2018.

GASPAR, M. et al. Gestão do conhecimento em empresas atuantes na indústria de software no Brasil: um estudo das práticas e ferramentas utilizadas. Informação \& Sociedade, v. 26, n. I, p. |5 |- |66, 2016.

GEREFFI, G. Global value chains in a post-Washington consensus 
world. Review of International Political Economy, v. 21 , p. 9-37, 2014.

GUERCI, M. et al. Moving beyond the link between HRM and economic performance: A study on the individual reactions of $H R$ managers and professionals to sustainable HRM. Journal of Business Ethics, I- I8, 2018.

HAHN, R.; KÜHNEN, M. Determinants of sustainability reporting: $A$ review of results, trends, theory, and opportunities in an expanding field of research. Journal of Cleaner Production, v. 59, p. 5-2I, 2013.

HAHN, I. S.; SCHERER, F. L.; OLIVEIRA, M. C. S. F. Relacionamento business-to-business em empresas varejistas de materiais de construção. Reuna, v. 21 , n. 3, p. 25-50, 2016.

KAROLCZAK, M. E.; SOUZA,Y.S. Recursos humanos para a economia do conhecimento na ótica da teoria do capital humano. Revista Alcance, v. 24, n. I, p. 66-80, 2017. KPMG. The KPMG survey of corporate responsibility reporting 2017. 2017. Disponível em: <http://www.kpmg.com/crreporting>. Acesso em: 12 set. 2019.

LELEBINA, O.; GAND, S. Beyond "Hero-based" management: revisiting HRM practices for managing collective expertise. Relations Industrielles, v. 73, n. I, p. 39-66, 2018.

MAMEDE, S. P. N. et al. Empresas brasileiras estatais e não estatais: uma análise das relações de endivida- mento. Revista Evidenciação Contábil \& Finanças, v. 2, n. 5, p. 4-22, 2017.

MANZANEQUE, M.; MERINO, E.; BANEGAS R. Corporate governance issues and director compensation structure in Spanish companies. African Journal of Business Management, v. 5, n. 22, p. 9164-9179, 20II.

MASCENA, M. C.; BARAKAT, S. R.; FISCHMANN, A. A. Relação entre características do conselho de administração e monitoramento. Revista Adm. Faces, v. 4, n. I5, p. I27-|43, 2016.

OLIVEIRA, J. M. et al. Gestão de pessoas e sustentabilidade: construindo caminhos por meio das práticas de capacitação. Rev. Adm. UFSM, v. 10, Edição Especial, p. I08-|26, 2017.

PETRY, J. F. et al. Influência da internacionalização das empresas brasileiras na criação de valor. Revista Eletrônica de Estratégia \& Negócios, v. I, n. 7, p. 158-180, 2014.

POZAS, O. H.; JAUREGUI, L. A snapshot of training practices in Peru. Estudios Gerenciales, v. 28, n. I24, p. 67-85, 2012.

ROBERTS, J. No one is perfect:The limits of transparency and an ethic for 'intelligent' accountability. Accounting, Organizations and Society, v. 34, p. 957-970, 2009.

ROSOLEN, T.; MACLENNAN, $M$. Strategic human resource management and corporate social responsibility: evidence from emerging markets. Internext, v. I I, n.
2, p. 66-80, 2016.

SCHALTEGGER, S.; WAGNER, M. Integrative management of sustainability performance, measurement and reporting. International Journal of Accounting, Auditing and Performance Evaluation, v. 3, p. I- 19, 2006.

SCHULTZ, T.W. Investment in human capital. The American Economic Review, v. 5I, n. I, p. I-I7, 1961.

SHRM. Advancing sustainability: HR's role. Society for Human Resource Management, BSR and Aurosoya SHRM, 201 I.

SILVEIRA, V. N. S.; MAESTRO FILHO, A. D. Gestão estratégica de pessoas e desempenho organizacional. Pretexto, v. I4, n. I, p. 7|-87, 2013.

SUSSLAND,W.A.The board of directors: a referee or a coach? Corporate Governance, v. 5, n. I, p. 65-72, 2005.

TASCA, J. E.; ENSSLIN, L.; ENSSLIN, S. R. A avaliação de programas de capacitação: um estudo de caso na administração pública. Revista de Administração Pública, v. 46, n. 3, p. 647-675, 2012.

UTRILLA, P. N.-C.; GRANDE-TORRALEJA, F. A. The human resources development through mentoring:The Spanish case. Intangible Capital, v. 8, n. I, p. 6I-9I, 2012.

UTRILLA, P. N.-C.; GRANDE-TORRALEJA, F. A.; LORENZO, D. The effects of coaching in employees and organizational performance: The Spanish case. Intangible Capital, v. I I, n. 2, p. I66-I89, 20 I5. 